\title{
Establishing a metrology lab in space to meet the demands of climate change
}

\author{
Nigel Fox ${ }^{1}$, Paul Green ${ }^{1}$, Rainer Winkler ${ }^{1}$ and Dan Lobb² \\ ${ }^{1}$ National Physical Laboratory, Hampton Rd, Teddington, Middx, TW11 OLW, UK \\ ${ }^{2}$ Surrey Satellite Technologies Ltd, Rayleigh House, 1 Bat and Ball Rd, Sevenoaks, Kent, TN14 5LJ, UK
}

\begin{abstract}
Le climat de la terre change, mais le taux de changement et ses effets indirects est loin de comprendre et largement débattu. Écarts de jusqu'à $5^{\circ} \mathrm{c}$ peuvent être vu entre les modèles de prévision climatique rend difficile pour les responsables politiques à prendre les mesures nécessaires pour atténuer et s'adapter à un réchauffement de la planète terre. Les observations globales de la terre par satellite sont le seul moyen de fournir la date nécessaire pour améliorer la fidélité des prévisions et en particulier de contraindre et de les tester. Ces données doivent être collectées décennies permettant aux petites tendances à être détecté issu d'un milieu de variabilité naturelle-mise des exigences sévères sur la performance des capteurs satellite. À présent peu si les satellites sont en mesure de livrer vraiment mesures traçables SI de l'orbite et zéro avec l'incertitude nécessaires pour les études climatiques à long terme, qui est proche de celle uniquement réalisables à l'aide d'étalons primaires en laboratoire. Le livre décrit une mission proposée par satellite conçue pour tirer le prima...
\end{abstract}

\section{Introduction}

\subsection{Background}

Unequivocal attribution of the causes, consequential impact and effective mitigation/adaptation of change in the Earth's climate is arguably the greatest challenge facing science and society today. The ongoing challenges stemming from the "Kyoto protocol", "Copenhagen accord" and hopefully culminating in the Conference of the Parties (COP 21 in Paris 2015, exemplify the intense political, scientific and public debate that is associated with the issue. This is underpinned by the enormous cost implications of policy decisions based on forecasted impacts. However these forecasts have significant uncertainty with estimates of global temperature increases ranging from at best, 2 to $5^{\circ} \mathrm{C}$ by 2100 see figure 1 , [1].

Such a range could be the difference between the need for major new flood defences to prevent large land losses or maintenance/minor updates of the status quo. These temperatures are global averages and both the local value of temperature and its impacts are anticipated to vary widely across the globe, with developing nations likely to be in regions most significantly affected. It is the duty of the science community to reduce this unacceptably large uncertainty in forecasts by finding and delivering the necessary information, with the highest possible confidence, in the shortest possible time.

The Intergovernmental Panel on Climate Change (IPCC) concludes that recent decades have revealed clear hallmarks of anthropogenic climate change, but the mix of natural variability and anthropogenic effects on decadal

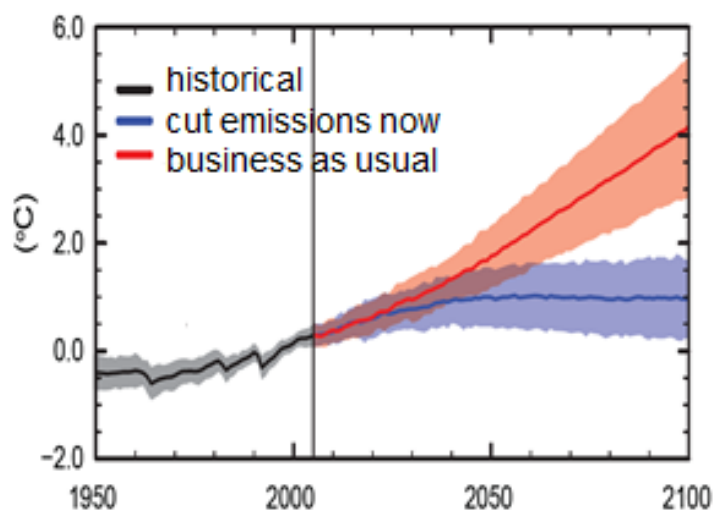

Figure 1 The uncertainty range in forecast temperatures of the Earth for two extreme emissions scenarios [1]

timescales is far from fully understood or measured, requiring significant improvements in accuracy [1].

Unequivocal attribution and the quantification of subtle fingerprint indicators is fundamental to our ability to reliably predict impact and the development of appropriate mitigation/adaptation strategies. The uncertainty in climate prediction lies in the complexity of the models, our inadequate understanding of the Earth system and its feedback mechanisms and the relatively poor quality of available data against which to test predictions on the necessary decadal timescales [2].

The data needed to benchmark and test these models must be global in nature, and collected over a sufficiently long-time base with appropriate uncertainty to enable the 
identification of "change" in a particular measurand above variance that could be caused by natural or "local" effects. In general this means that such measurements need to be considered on decadal timescales and collected from satellite based instrumentation.

Terrestrial networks, collecting "in-situ" data, will always be necessary to provide local validation of global observations and support detailed studies of Earth system processes e.g. air quality, carbon and hydrological cycles etc. However, it is only remote and continuous observation of the Earth system by satellite that the key data sets, the Essential Climate Variables (ECVs) identified by the Global Climate Observing System (GCOS) of the United Nations [3], can ultimately be adequately addressed. In general, these ECVs (e.g. albedo, cloud cover, chlorophyll-a ...) are not measured directly but derived from more fundamental and familiar physical quantities such as: radiances, reflectance, transmittance etc often now referred to as Fundamental Climate Data Records (FCDR). The satellite instruments measuring these quantities utilise the full electromagnetic spectrum and in most cases passively, for example making use of the sun as a source for reflectance/radiance/transmittance measurements.

A community workshop held in the USA in 2007, considered the needs of climate $[4,5,6]$ and concluded that in the optical domain, the critical underpinning measurands (FCDRs) and the associated uncertainty requirements were:

- Total Solar Irradiance (TSI) $0.01 \%(\mathrm{k}=1)$

- Solar Spectral Irradiance (SSI) $0.1 \% \quad(\mathrm{k}=1)$

- Earth Reflected solar radiance $0.3 \% \quad(\mathrm{k}=2)$

- Earth Emitted Infrared (IR) radiances $0.1 \mathrm{~K}(\mathrm{k}=3)$

(Expressed in terms of resultant temperature)

$\mathrm{Nb}$ spectral resolution for SSI needs to be 1 to $5 \mathrm{~nm}$ (ideally from 200 to $2500 \mathrm{~nm}$ ) and for Earth spectral radiance $<\sim 20 \mathrm{~nm}$ from 320 to $2400 \mathrm{~nm}$.

This, and their priority, was reinforced in the decadal review carried out by the National Research Council of USA [7].

Sound policy making requires high confidence in climate predictions verified against decadal change observations with robustly known accuracy. Yet, our ability to monitor and predict the future of the climate is inadequate as we currently do not possess sufficient accuracy in our observing capability to confidently observe the small but critical climate change signals that are expected to occur over decadal time scales. We currently rely on data from sensors primarily designed for operational weather forecasting, where short to medium term global consistency and continuity is of greater importance than absolute accuracy and long-term continuity is achieved largely from overlapping and normalisation of data sets. It is now increasingly recognised by the community that this is inadequate and that the robustness and long term confidence stemming from SI traceability and the move towards a global climate observing system needs to become a higher priority [8].

In practise this means there is a need to dramatically improve the absolute accuracy of in-orbit observation (factor of ten) if we wish to maximise our sensitivity to critical climate signals, mitigate the risks of gaps in the data record and provide the observations necessary to evaluate the accuracy of climate model predictions. To address this lack in our observing capability three critical measurements and their required accuracies were defined by the US National Research Council Decadal Survey, one of which was Earth reflected solar spectral radiation [7].

\subsection{Key climate parameters.}

Uncertainties in climate feedbacks, which are key to determine how the climate system will respond to a given forcing, are the primary cause for our current uncertainty in climate prediction. The most important of these in order of uncertainty in the magnitude of their contribution are: cloud feedback, temperature lapse rate/water vapour feedback, and snow/ice albedo feedback [1]. Understanding how these aspects of the climate system respond and evaluating the fidelity of the response in climate models is essential if we are to improve our understanding of how the climate will change and thus take action in a timely manner.

Solar radiation, reflected from the Earth-atmosphere system back to space, constitutes a powerful and highly variable forcing of the climate system through changes in snow cover, sea ice, land use, cloud and aerosol properties. Systematic, spatially resolved observations of the time series of the absolute spectrally resolved flux of near-ultraviolet, visible, and short wave-IR radiation returned to space by the Earth system, tied to international standards in perpetuity, underpin a credible climate record of the changing Earth system.

In combination with establishment of the absolute spectrally resolved solar irradiance reflected from the Earth-atmosphere system to space, to address the above, it is essential to continue the long-term, time series of incident total solar irradiance (TSI), and enhance its climate value through improved accuracy, removing the current controversies and uncertainties due to instrumental variations and their linkage into a climate record. The addition of a high accuracy spectrally resolved measurement of incoming solar irradiance, provides the means to link solar variation to climate processes: underpinning studies of atmospheric chemistry and facilitating retrieval of the full range of Earth system products at both top and bottom of atmosphere.

At present there is no planned (funded) satellite mission capable of meeting these observation requirements. However, there are two complimentary mission which have been proposed that can deliver this step change in performance and meet the challenge which is primarily metrological in nature. One is the NASA CLARREO mission [9] and the other, which is the subject 
of this paper, is the European TRUTHS mission led by NPL. $[10,11]$

\section{Traceable Radiometry Underpinning Terrestrial- and Helio- Studies (TRUTHS): an NMI in space}

\subsection{Introduction}

The overall requirement driving the specification of TRUTHS can be summarised as: a satellite mission to determine the state of the climate (in the solar reflective spectral domain) as a benchmark reference of sufficient SI-traceable accuracy to enable trends to be detected in the shortest time possible, limited only by noise from natural variability of the Earth System. In essence to achieve the objectives set out in section 1 above.

In achieving this it will also significantly enhance the performance and traceability of other Earth observing systems, for example the sentinels of EU/ESA Copernicus program, enabling them to deliver additional societal benefits and, in some cases, "climate quality" data products, from existing instruments. In fulfilling these objectives it will provide the key building block to initiate a global climate observatory from space.

TRUTHS will achieve these objectives by two independent but complimentary methodologies:

- Direct sampling of key climate specific fingerprint signatures as baselines from which future change can be detected.

- Facilitating performance improvements in other observing systems, satellites and others to enable them to collect better "climate quality" data through reference calibrations.

These objectives drive the specification of the mission- the top three bullets in section 1.1, globally and continuously sampled with a spatial resolution on the Earths surface at nadir of $50 \mathrm{~m}$. (note the spatial resolution is not driven by climate benchmark measurements themselves, which only require zonal averages, but rather to ensure a good match to the footprint of other sensors being calibrated). The data stemming from these driving requirements can of course have many other applications e.g. agriculture, forests, resource monitoring, in common with other satellites, but delivered with significantly higher accuracy and confidence. Although demanding, the underpinning observation technology and satellite platform are largely developed and already proven as space instrumentation. The innovation derives from the paradigm shift in the way it is used and in particular the disruptive nature of the means to achieve SI-traceable onboard calibration.

TRUTHS will fly the radiometric capability (including the primary standard) of a national measurement institute (NMI) into space. In this way it will be able to act like a calibration or "standards laboratory in space" - TRUTHS will be SI-traceable by design. Thus, this paper will not dwell on the details of the instrumentation, only seek to highlight any key characteristics to aid the reader understand the concepts.

The key challenge to this mission and thus one where we will concentrate our efforts in terms of explanation is the ability to achieve SI traceability at uncertainty levels a factor of 10 below what has been achieved to-date. We will seek to show how this step change is not in practice as radical as it might initially appear but rather the result of taking the terrestrial laboratory calibration chain directly into orbit. In fact, when the methodology which will be described here was first introduced into NMIs, some 30 years ago, a step change of $>50$ was achieved.

TRUTHS is implemented through a small agile satellite platform with two perpendicular observation axis: Solar and Earth viewing see Figure 2. The majority of observation time is allocated to global nadir spectrally and spatially resolved measurements of Earth reflected solar radiation for decadal climate change benchmarking. A nominal 5 to 10 minutes per day is allocated to solar observations (total and spectrally resolved). In addition, the agile platform enables:

- near simultaneous angularly co-aligned observations for sensor reference calibrations,

- multi-angular measurements of specific targets for reference inter-calibration of sensors (surface and moon)

- Multi-angular measurements of specific targets to support 'process studies'.

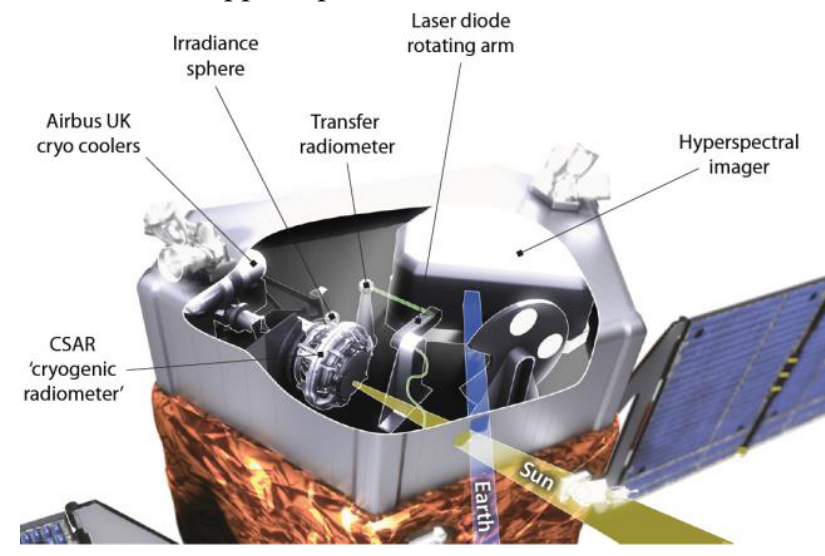

Figure 2: Schematic representation of the TRUTHS payload and its operational configuration. The satellite has two viewing directions, towards the Sun or Earth, shown here approximately 60 degrees to each other.

The very high radiometric accuracy required by TRUTHS means that it seeks to be largely self-reliant in most of its key measurands and thus includes the capability required to provide the information to retrieve the atmospheric parameters (e.g. aerosols, water vapour) required to account for radiation propagation losses in the atmosphere.

\section{TRUTHS instrumentation}

A full description of the instrumentation employed on TRUTHS is beyond the scope of this paper but a brief summary of the key instrument is included here to aid the 
in the outline description of the novel calibration method, more details can be found elsewhere [10,11].

\subsection{Cryogenic solar absolute radiometer (CSAR)}

The Cryogenic solar absolute radiometer (CSAR) is the heart of the TRUTHS mission [10], providing the means to establish SI traceability in orbit. CSAR (and laboratory cryogenic radiometers) are based on the principle of 'electrical substitution', where optical power incident on a black (spectrally neutral) absorbing surface will cause a rise in temperature of the disk, which is sensed by a thermometer. With the optical power shut off, the same temperature rise is then established by passing a current through an electrical heater attached to the disk. The electrical power used to create that temperature rise can be equated to the optical power, and since electrical power can be measured with relative ease, the optical power can be determined through the substitution of electrical power.

Although similar instruments are already used in space to measure TSI, this mission will be the first to fly such an instrument, cooled to cryogenic $(\sim 20 \mathrm{~K})$ temperatures, using an Airbus cooler. Cooling such instruments in terrestrial laboratories $\sim 30$ years ago led to a step change reduction in radiometric uncertainties of nearly a factor of 100 and are now the primary standard of choice at most of the World's NMIs. CSAR will be the primary standard providing SI traceability for the calibration of all on-board optical instrumentation. This standard will provide traceability to SI to an absolute accuracy of $0.1 \%(\mathrm{k}=2)$.

In addition to serving as a primary standard for radiance and irradiance, CSAR will also provide measurements in its own right:-Total Solar Irradiance (TSI) at an uncertainty of $0.02 \%(\mathrm{k}=2)$, a factor of ten better than the currently operational ambient temperature radiometers.

\subsection{In-flight calibration method}

The calibration method for the TRUTHS Earth Imager (EI) has simplified from that originally described in [10] and is now a three step process. The SI primary standard 'traceable' cryogenic radiometer (CR) power measurement capability is transferred via a monochromatic based source to a simple transfer radiometer (TR) and from there, using the same source to provide a known radiance field to over-fill the entrance aperture of the EI via a Lambertian diffuser plate. The stepwise process is shown schematically in Fig. 3.

It should be noted that in an attempt to make clear the basic steps the schematic representations are not necessarily intended to represent a real or even close to real layout, only the concept. For example, in some cases there may be additional turning mirrors or optical elements to help practical engineering implementations but it should be noted that these will not impact the overall traceability concept or its ability to achieve the required uncertainties.

\subsubsection{Step 1:}

A stabilised (temperature and current control) suite of low power laser diodes (LD) is used as a source, its power measured by the cryogenic radiometer (CSAR) and then used to calibrate the response (to power) of the TR, underfilling its two field of view (FOV) defining apertures. (The TR is a small integrating sphere, with integral semiconductor photodiodes, Silicon and InGaAs, viewing the diffusely illuminated wall), removing any sensitivity due to alignment.

This step requires the LD output to be stable in wavelength and intensity during the time period for the measurement $<1$ minute. Studies indicate this is readily achievable.

The output of the LD is moved between the two instruments using for example, a rotating mirror assembly imaging the output of an optical mixing rod used to coalign the outputs of the LDs.

The result of step 1 is a TR calibrated for spectral response (and spectral radiance response, through knowledge of the FOV geometry) for a discrete set of monochromatic wavelengths.

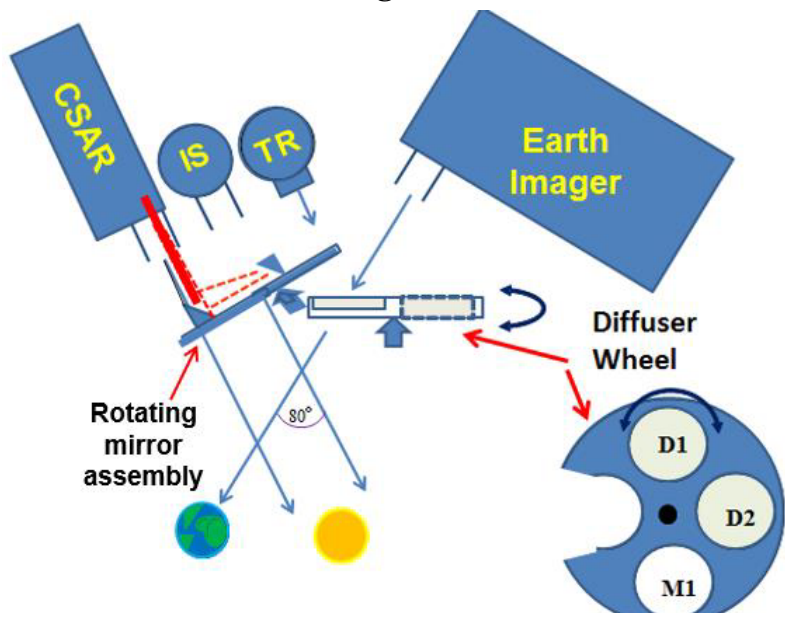

Figure 3a: Laser power measured by CSAR (Step 1)

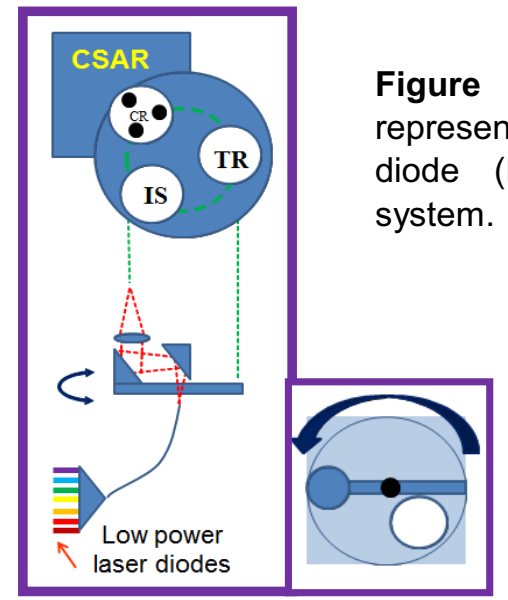




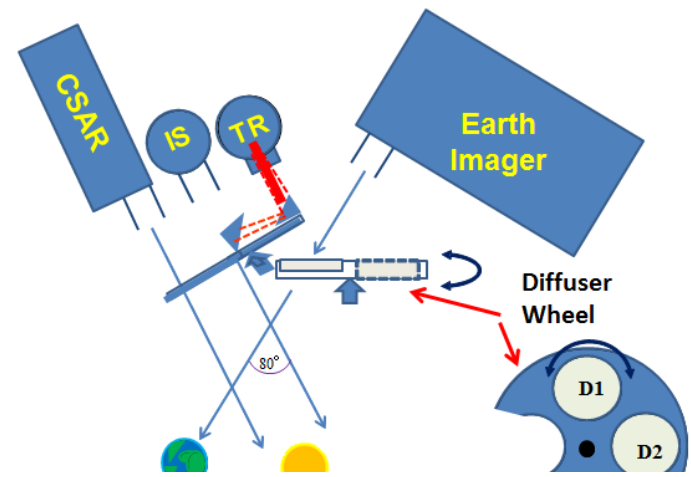

Figure 3c. Mirror assembly rotates to allow LD radiation to calibrate response of TR (Step 1)

\subsubsection{Step 2:}

The LD output is expanded to create a uniform illumination of a flat plate lambertian diffuser (mounted on a rotation wheel to allow it to be moved in and out of the FOV of the EI), providing a radiance source which can be viewed by the TR and the EI (following rotation of the diffuser). This is achieved by under-filling the input aperture of the irradiance sphere (IS), which provides a convenient means to homogenise and uniformly expand the LD beam, and then using a mirror to illuminate the exit port on to the diffuser.

The previous measurement in step 1 , together with knowledge of the geometry of the TR two FOV defining apertures allows the TR to measure the reflected monochromatic radiance of the diffuser plate. The diffuser now provides a known source of radiance, to calibrate the EI at each of the LD wavelengths.

An independent measure of the IS throughput is not needed, just short-term (seconds) stability of the LD \& IS combined system and consistency of the LD wavelength used in step 1.

The result of step 2 is the EI calibrated for spectral radiance at a number (typically ten) of discrete monochromatic wavelengths across its spectral range, directly traceable to the CSAR.

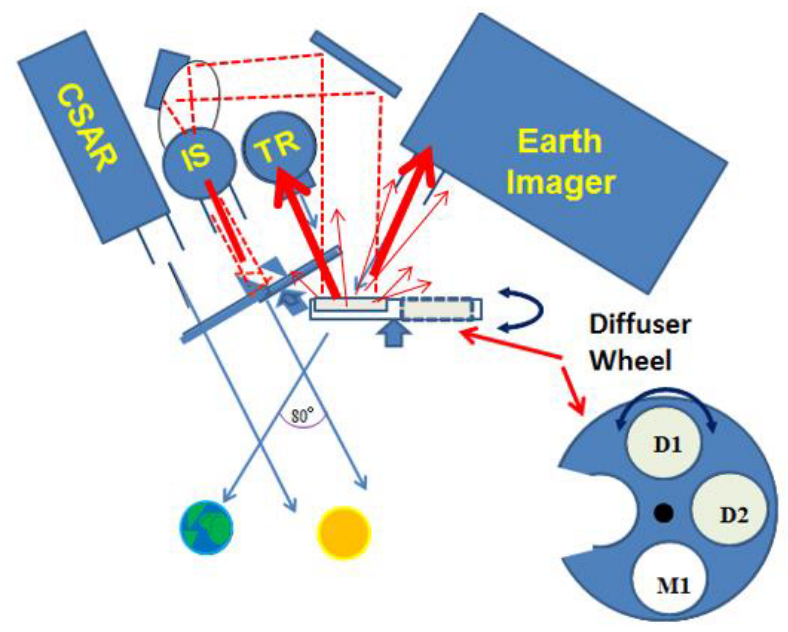

Figure 3d. mirror assembly rotates to allow LD to irradiate IS which then illuminates diffuser (Step 2)

\subsubsection{Step 3:}

An incandescent lamp (with a smooth spectral output) illuminates the IS and consequently the diffuser and is viewed by the EI. This provides a means to interpolate the spectral radiance calibration between the LD wavelengths.

Although the lamp output will change, this will largely be in terms of absolute level and/or relatively smooth and small spectral variation. The absolute level and spectral shape is anchored by the LD calibration points on the EI.

It should be noted that the TR and diffuser used in steps 1-3 never need to be exposed directly to sunlight, thus limiting the solarisation degradation of these components.

The result of this step is a fully spectrally radiometrically calibrated $E I$ which can observe the Earth.

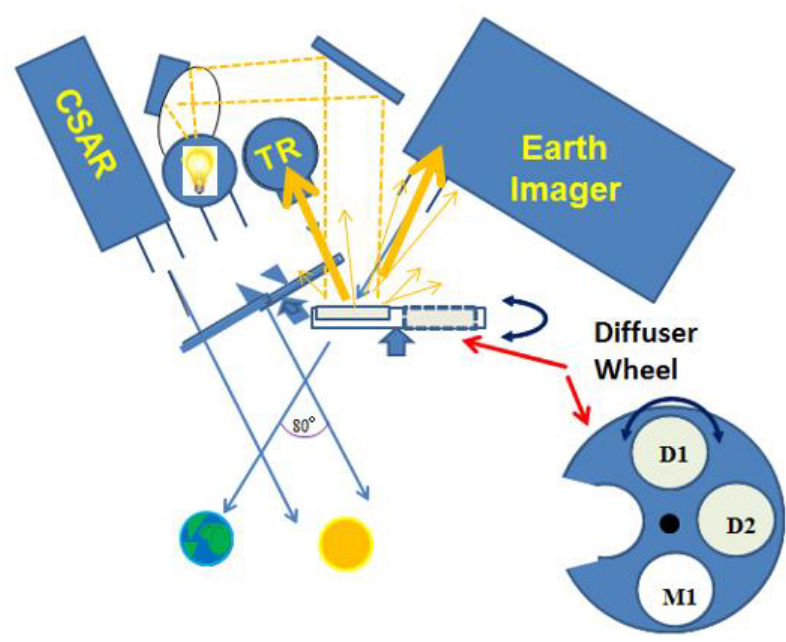

Figure 3e. Lamp illuminates IS and then diffuser to allow spectral interpolation (Step 3)

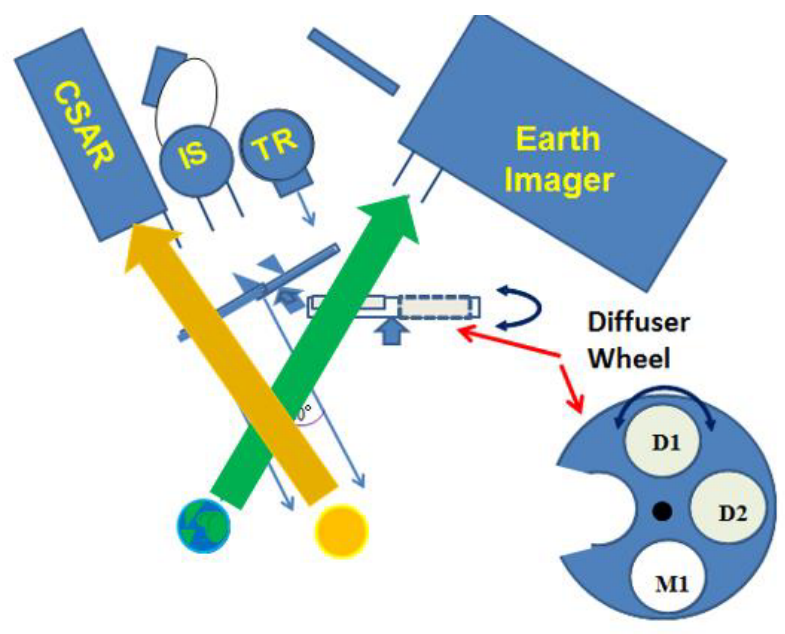

Figure $3 f$ Diffuser rotates to allow El and CSAR clear view of the Earth and Sun respectively

Figure 3 (a-e).Schematic representation of the in-flight calibration method of TRUTHS. 
A similar set of steps can be shown for the use and calibration of the EI for measuring solar spectral irradiance.

\section{Conclusions}

This paper has outlined the need and urgency for significantly improved accuracy in measurements made of the Earth-Sun system to provide policy makers and society the unequivocal evidence required to ensure concerted timely action from the worlds governments.

The necessary improvements in accuracy present a grand challenge to the metrology community to provide the methods/standards to facilitate a step change in uncertainty, in space, to that comparable to what is currently achieved using primary standards in laboratory conditions.

The paper describes how this grand challenge has been embraced by the metrology community resulting in a small satellite mission proposal, TRUTHS, to establish an NMI in space. The underpinning metrological concepts of how SI traceability can be established on-board the spacecraft have been described. Although the mission is not as yet funded it and its sister CLARREO, are receiving significant international attention and have been through initial engineering studies in preparation for space implementation. TRUTHS for example could be ready for launch within 3 to 4 years.

Of particular interest is the ability for the missions to upgrade the performance of other satellite observing systems through reference calibration, in this way enabling the establishment of a global climate observing system in the relative near term without the significant cost of many new specifically tailored satellites.

In summary, the urgency of a climate benchmark mission is such that early implementation is imperative and TRUTHS offers a means to achieve that for solar spectral domain and in doing so provides a means to upgrade the performance of the whole Earth Observing system and provide a showcase example of the benefits of metrology and SI traceability.

\section{References}

1 IPCC, 2013: Fifth Assessment Report of the Intergovernmental Panel on Climate Change, in: Climate Change 2007: The Physical Science Basis https://www.ipcc.ch/report/ar5/wg1/

2 K.E,.Trenberth, and J. T. Fasullo, CLIMATE CHANGE Tracking Earth's Energy, Science, 328 (5976), 316-317 (2010).

3.

Http://www.wmo.int/pages/prog/gcos/index.php?na me=EssentialClimateVariables

4 G. Ohring (Ed), Achieving Satellite Instrument Calibration for Climate Change (ASIC3), NPOESS Workshop Report, 144. (2007)

5

https://www.wmo.int/pages/prog/gcos/Publications/gcos107.pdf. (2006)
6 G.B. Ohring,, B. A. Wielicki, R. Spencer, B. Emery and R. Datla, Bulletin of the American Meteorological Society, 86 1303-1313 (2005).

7 NRC, 2007: Earth Science and Applications from Space: National Imperatives for the Next Decade and Beyond, $418 \mathrm{pp}$.

8 http://www.wmo.int/pages/prog/sat/documents/ARC H_strategy-climate-architecture-space.pdf

9 B. Wielicki et al, Bull. Amer. Meteor Soc., 95, 1519$1539,(2013)$

10. N.P. Fox, Kaiser-Weiss, A., Schmutz, W., Thome, K., Young, D., Wielicki, B., Woolliams, E. Philosophical transactions. Series A, Mathematical, physical, and engineeringsciences,369, 4028-63. (2011).

11. http://www.npl.co.uk/truths

\section{Acknowledgments}

We thank UK Space Agency through its Centre for Earth Observation Instrumentation (CEOI) and the European Metrology Research Program (EMRP) of the European Union and EURAMET, together with the National Measurement Office of the Business Innovation and Skills (BIS) of UK government for their financial support. 